\title{
Challenges in the management of aortic coarctation in the elderly: native coarctation complicated by severe calcification
}

Edgar LW $\underline{\text { Tay }}^{1,2}$, MBBs, Namal Wijesinghe ${ }^{1}$, MD, Jonathan Avrom Leipsic ${ }^{1}$, MD, Ronald Carere $^{1}$, MD

ABSTRACT Although the treatment of aortic coarctation in adults with the use of stents has shown favourable results and reduced complications, there have been few studies involving elderly patients. We highlight the clinical challenges faced in the management of such patients, with attention to severe calcification at the coarctation site. The midterm results were good in our patient, showing an improvement in blood pressure control and maintenance of stent patency.

Keywords: adult congenital heart, coarctation, hypertension

Singapore Med J 2013; 54(1): e5-e8

\section{INTRODUCTION}

Compared to the use of balloon angioplasty alone, endovascular treatment of aortic coarctation in adults with the use of stents has shown favourable results and a reduction in complications. (1) Thanopoulos et al recently reported sustained results of up to five years in selected adult patients. ${ }^{(2)}$ However, few studies have addressed the difficulties in the management of older patients with coarctation who are likely to have advanced disease with complications such as hypertension and concomitant coronary artery disease, and who are more likely to be at risk for inferior outcomes following treatment. In addition, unfavourable anatomy in these patients may hinder successful surgical or percutaneous management.

\section{CASE REPORT}

A 65-year-old Caucasian woman with a history of coarctation of the aorta presented in December 2007 with chest and shoulder pain associated with significant elevation of troponin I level, and was diagnosed with non-ST-segment elevation myocardial infarction (NSTEMI). She had not been previously told that she needed treatment for coarctation and was thus not on regular follow-up. Her left arm blood pressure was 180/90 $\mathrm{mmHg}$ and she had a grade 3 ejection systolic murmur heard over the aortic region, which radiated posteriorly. Lower limb pulses were not palpable. Despite treatment with atenolol $100 \mathrm{mg}$, losartan $100 \mathrm{mg}$, frusemide $40 \mathrm{mg}$ and methyldopa $500 \mathrm{mg}$ twice daily, her systolic blood pressure remained persistently elevated. Cardiac catheterisation following this acute presentation showed normal coronaries, but a significant peak-to-peak gradient of $60 \mathrm{mmHg}$ across a highly calcified coarctation site was found.

The patient had undergone multiple orthopaedic surgeries on both knees as well as repeated surgeries for iritis, one of which was complicated and required enucleation of the left eye. She also had severe subcapsular cataracts from chronic steroid

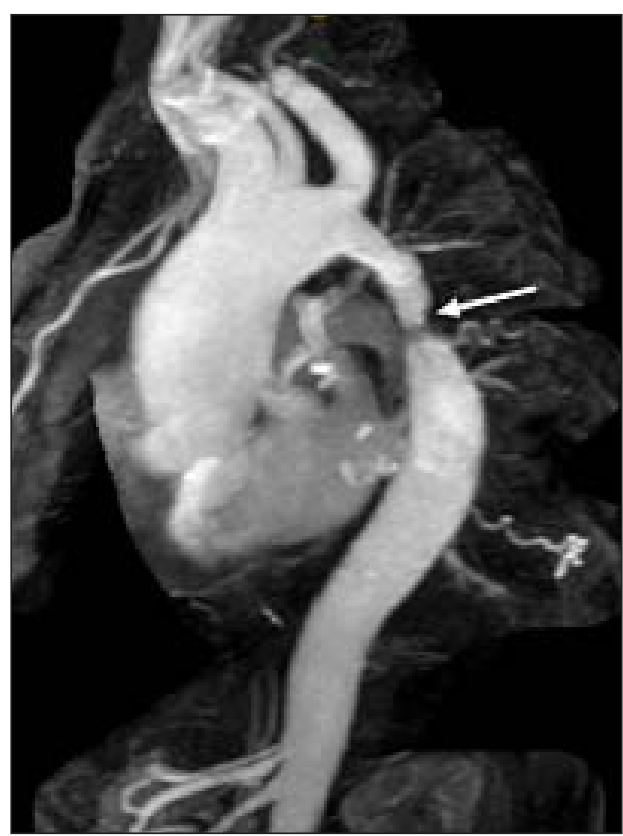

Fig. 1 MR angiography with gadolinium shows the coarctation site (white arrow).

therapy for her joint pains. Her past medical history includes a right carotid to right subclavian bypass in 1994 (no details were available), thyroidectomy for papillary carcinoma and treated pulmonary tuberculosis.

Transthoracic echocardiogram revealed a trileaflet aortic valve with features of concentric left ventricular hypertrophy and preserved ventricular ejection fraction. Cardiac magnetic resonance (MR) imaging and computed tomography (CT) confirmed the diagnosis of coarctation, showing a long and calcified lesion (Figs. 1 \& 2) with a minimum diameter of $4 \mathrm{~mm}$. This case was extensively discussed among congenital cardiologists and cardiac surgeons, and it was decided that percutaneous treatment was indicated, given the severity of the lesion, poorly controlled hypertension and high surgical risk. However, the

${ }^{1}$ St Paul's Hospital, University of British Columbia, Vancouver, Canada, ${ }^{2}$ National University Health System, Singapore

Correspondence: Dr Edgar Tay, Consultant Cardiologist, NUHS Tower Block Level 9, 1E Kent Ridge Road, Singapore 119228. Edgar_Tay@nuhs.edu.sg 


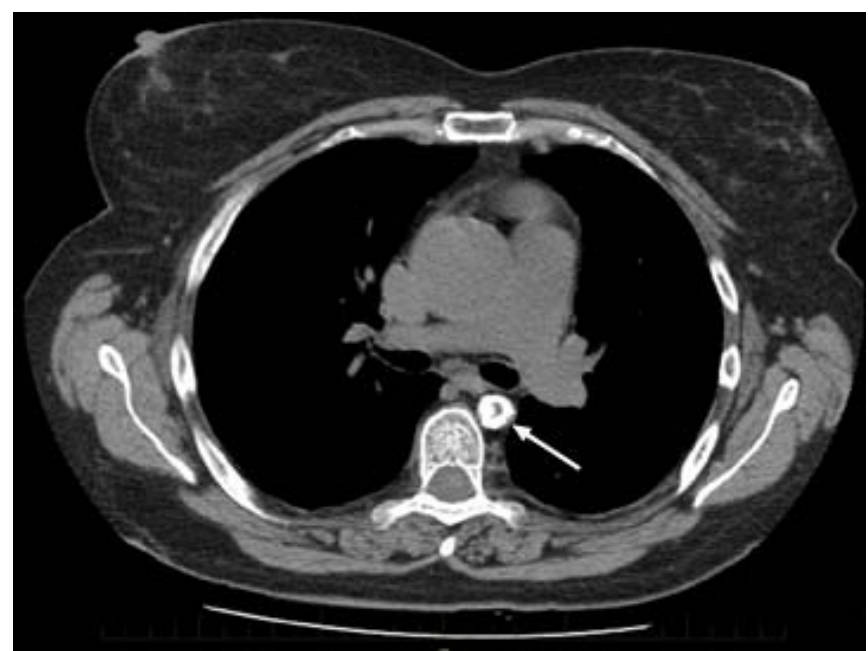

Fig. 2 Axial CT image shows the severe circumferential calcification and narrowing of the coarctation (white arrow).

patient re-presented prior to the procedure with another similar episode of NSTEMI, with a maximum troponin level of $12 \mathrm{ug} / \mathrm{L}$. Coronary angiogram again showed no significant coronary artery disease. It was speculated that severe hypertensive episodes could account for these events. However, it was noted that her blood pressure recorded at presentation (albeit delayed) in the emergency department was $170 \mathrm{mmHg}$.

The percutaneous procedure was performed following informed consent. A covered Cheatham-platinum stent ${ }^{\mathrm{TM}}$ was made available in the catheterisation laboratory and surgical backup was prearranged in the event of vascular complications. An $8 \mathrm{Fr}$ sheath was inserted into the right femoral vein to facilitate transvenous ventricular pacing. The right femoral artery was preclosed with a 6 Fr Perclose ProGlide. A 6 Fr sheath was initially inserted and a 6 Fr multipurpose catheter with a $0.038^{\prime \prime}$ Terumo angled Glidewire ${ }^{\circledR}$ was used to cross the coarctation. This was subsequently exchanged with a $260-\mathrm{cm} 0.035^{\prime \prime} \mathrm{J}$ wire with its distal tip positioned in the left brachial artery, due to the occlusion of the right subclavian artery. A peak-to-peak gradient of $70 \mathrm{mmHg}$ was obtained across the coarctation. Initial balloon angioplasty at $10 \mathrm{~atm}$ for ten seconds was performed with a $5 \mathrm{~mm} \times 40 \mathrm{~mm}$ Ultra-thin ${ }^{\mathrm{TM}}$ Diamond balloon (Fig. 3). No waist was seen on the balloon during this initial inflation. The arterial sheath was upsized to an $80-\mathrm{cm} 14 \mathrm{Fr}$ Check-Flo II Intro Set. Following this, a $10 \mathrm{~mm} \times 20 \mathrm{~mm}$ Ultra-thin ${ }^{\mathrm{TM}}$ Diamond balloon was introduced and sequentially inflated at 9-12 atm, at increments of $1 \mathrm{~atm}$. The peak-to-peak pressure fell to $35 \mathrm{mmHg}$, but a localised dissection was seen at the inferior border of the coarctation. A Palmaz XL 3110 stent was crimped onto a $12 \mathrm{~mm}$ $\times 40 \mathrm{~mm}$ Ultra-thin ${ }^{\mathrm{TM}}$ Diamond balloon. The $14 \mathrm{Fr}$ sheath was initially positioned proximal to the stenosis. The stent and balloon were then introduced within the sheath, followed by withdrawal of the sheath when a satisfactory position was achieved. With burst ventricular pacing at 180 beats per minute (to minimise cardiac output and potential stent displacement), the stent was deployed at $8 \mathrm{~atm}$ for ten seconds. A second inflation was performed at $10 \mathrm{~atm}$ for seven seconds, following which,
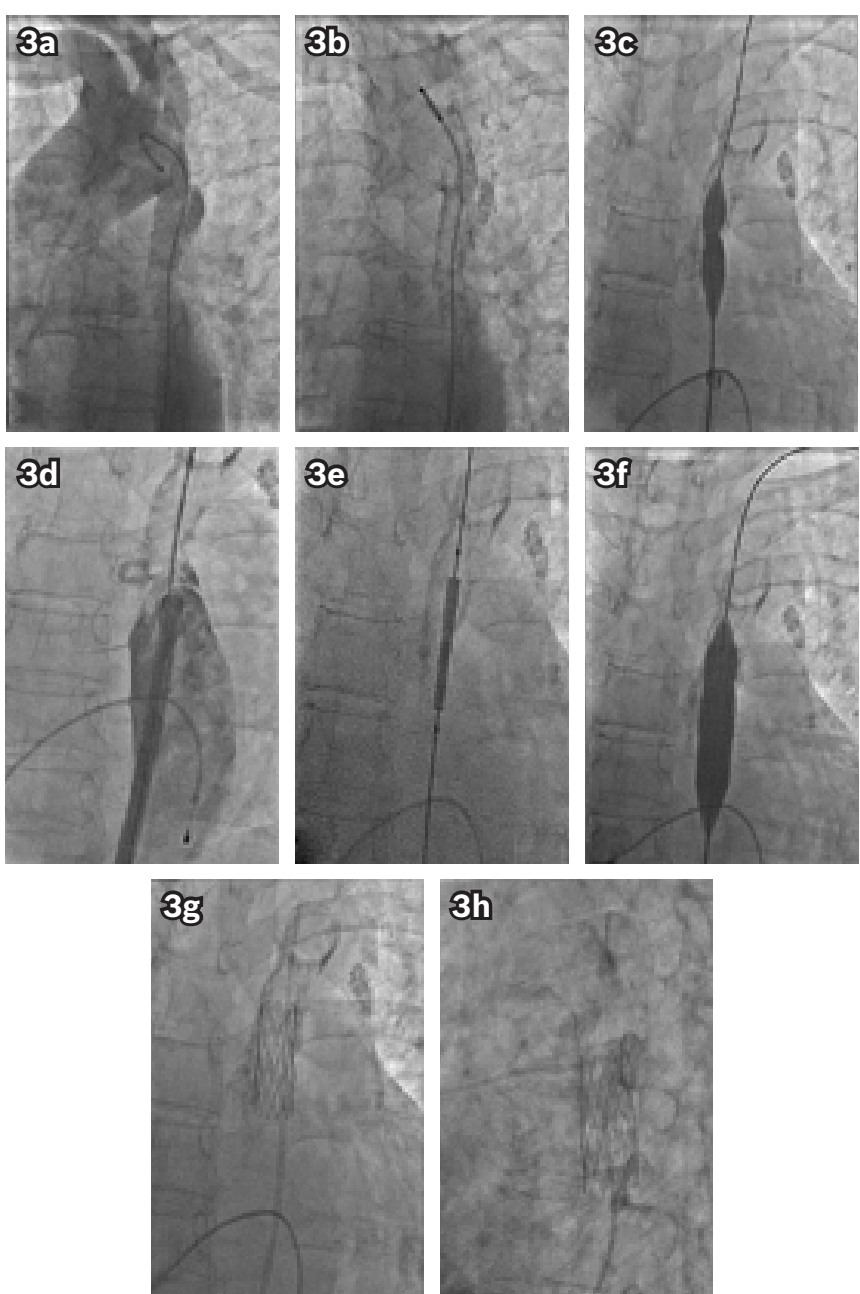

Fig. 3 Angiography images demonstrate the ( $a$ \& b) heavily calcified and long segment of stenosis; (c) waisting of the balloon during initial angioplasty; and (d) subsequent localised dissection seen in the inferior aspect of the coarctation. The stent is positioned (e) before (f) stent deployment. The final result shows good stent expansion. Note that the expansion is ( $g$ ) less in the anteroposterior, as compared to (h) the lateral projection.

the peak-to-peak gradient fell to $12 \mathrm{mmHg}$ (Fig. 4). Contrast angiography showed a good angiographic result with no evidence of further dissection or contrast extravasation. The patient was discharged uneventfully the following day with clopidogrel $75 \mathrm{mg}$ for a month, continued with $325 \mathrm{mg}$ of aspirin.

Six months after the procedure, her blood pressure control improved and her antihypertensives were reduced to two medications. Repeat CT imaging (ten months post-procedure) showed that the stent was widely patent, without evidence of neointimal hyperplasia or stenosis (Fig. 5).

\section{DISCUSSION}

Although it has been previously described, coarctation of the aorta presenting as acute coronary syndrome is rare..$^{(3,4)}$ This condition is associated with premature coronary artery disease, therefore a coronary angiogram would be indicated in such a presentation. ${ }^{(5)}$ Significant coronary artery disease was excluded in our patient, but it remains uncertain if the abovementioned events were related to episodes of coronary vasospasm or severe hypertension. 

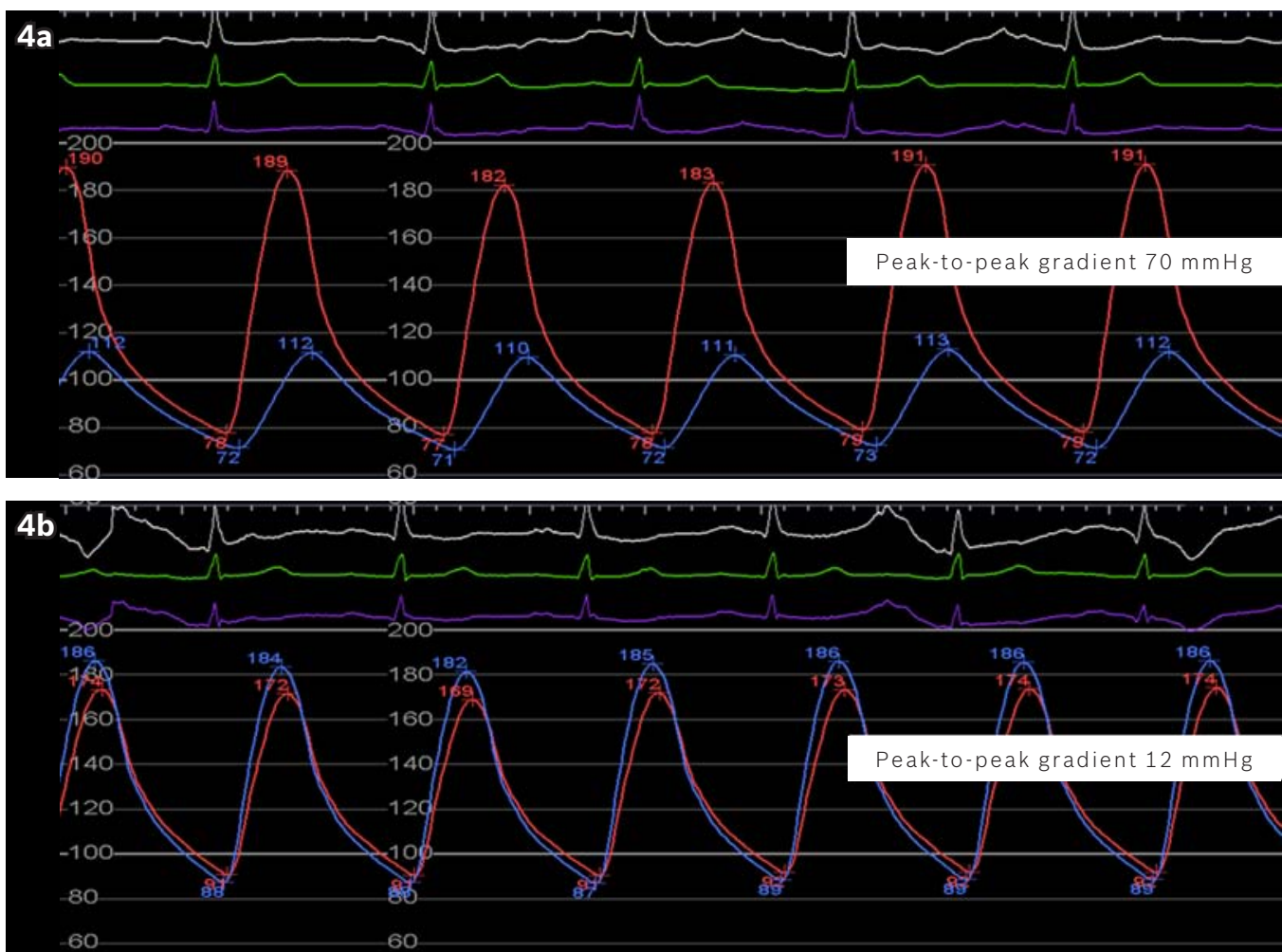

Fig. 4 Haemodynamics show (a) the marked gradient across the coarctation before stent implantation and (b) the final pressure gradient after stenting.

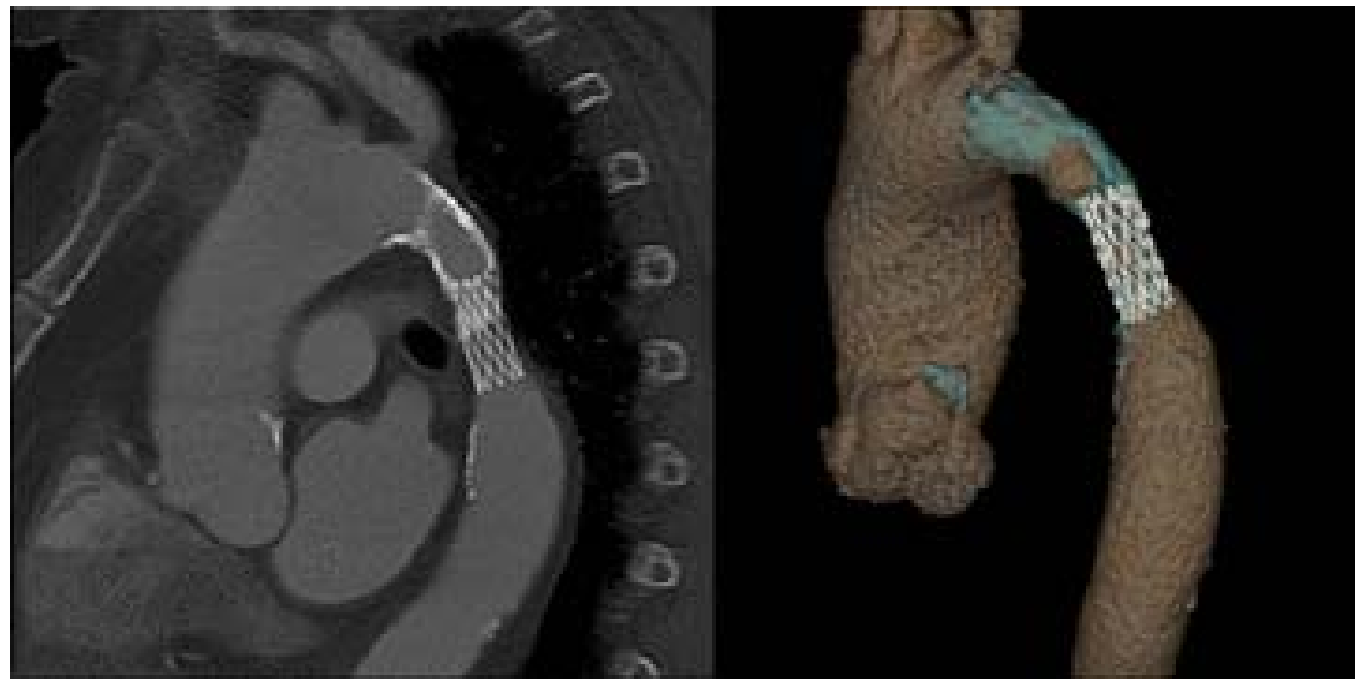

Fig. 5 CT images show sustained results at the ten-month follow-up.

This case demonstrates the challenges in the management of elderly patients. The therapeutic goal in this patient was to reduce the coarctation gradient in order to facilitate better blood pressure control, as well as reduce possible episodes of hypertensive crises and further myocardial injury. While current data suggest that the repair of coarctation of the aorta improves blood pressure control in many patients, ${ }^{(6,7)}$ some are left with significant hypertension, especially those treated late in life. However, most case series have demonstrated better blood pressure control with fewer medications needed in patients. ${ }^{(8)}$ Unfortunately, these studies are limited by small sample size and few of them addressed patients older than 60 years of age.
The presence of a highly calcified and long segment of stenosis also presents a technical challenge. Surgical treatment is challenging and a bypass procedure would be a consideration. With areas of circumferential calcification, as in this case, balloon dilatation may fail to achieve adequate expansion. There is also the risk of aortic rupture and dissection to contend with in this unique population with longstanding hypertension and associated atherosclerotic disease.

We initially planned to address our patient's lesion in a staged manner to reduce the risk of rupture with deliberate underdilatation of the coarctation (with or without stenting), followed by a second, more definitive procedure. The dissection following the 10-mm balloon dilatation led us to proceed with 
stenting. We chose a bare metal stent as our first approach, as we were uncertain if a covered stent could be expanded to the minimum recommended diameter of $12 \mathrm{~mm}$. The result with the Palmaz XL 3110 stent was angiographically and functionally very satisfactory, and we chose not to risk further expansion.

This case illustrates the percutaneous approach as a viable option for patients with aortic coarctation, even in the presence of severe calcification. The improvement in blood pressure control after treatment shows that elderly patients can benefit from this therapy. However, the treatment of these patients should be individualised and done in close consultation with a team of congenital cardiologists and surgeons. Covered stents should be made available in the event of an aortic rupture or severe dissection. Surgical backup should also be available during these procedures. Close follow-up would be mandatory in order to access the long-term outcomes of these procedures.

\section{REFERENCES}

1. Zabal C, Attie F, Rosas M, Buendía-Hernández A, García-Montes JA. The adult patient with native coarctation of the aorta: balloon angioplasty or primary stenting? Heart 2003; 89:77-83.

2. Thanopoulos BV, Eleftherakis N, Tzanos K, Skoularigis I, Triposkiadis F. Stent implantation for adult aortic coarctation. J Am Coll Cardiol 2008; 52:1815-6.

3. Takahashi Y, Takayama M, Sato N, et al. Restoration of endothelial function after repaired coarctation of the aorta--a case report. Angiology 2003; 54:609-11.

4. Yeşilay A, Topaloğlu S, Aras D, et al. A severe coarctation of the aorta incidentally diagnosed during cardiac catheterization of a 40-year-old male patient presenting acute coronary syndrome. Anadolu Kardiyol Derg 2007; 7:E1-2.

5. Vlodaver Z, Neufeld HN. The coronary arteries in coarctation of the aorta. Circulation 1968; 37:449-54.

6. Bhat MA, Neelakandhan KS, Unnikrishnan M, et al. Fate of hypertension after repair of coarctation of the aorta in adults. Br J Surg 2001; 88:536-8.

7. Wells WJ, Prendergast TW, Berdjis F, et al. Repair of coarctation of the aorta in adults: the fate of systolic hypertension. Ann Thorac Surg 1996; 61:1168-71.

8. Hashemzadeh K, Hashemzadeh S, Kakaei F. Repair of aortic coarctation in adults: the fate of hypertension. Asian Cardiovasc Thorac Ann 2008; $16: 11-5$ 